\title{
Two Novel Synchronized Bus-Clamping PWM Strategies Based on Space Vector Approach for High Power Drives
}

\author{
G. Narayanan, Member, IEEE, and V. T. Ranganathan, Senior Member, IEEE
}

\begin{abstract}
Two synchronized bus-clamping pulse width modulation (PWM) strategies based on the space vector approach are proposed for high-power induction motor drives. The two strategies together can produce PWM waveforms with any odd pulse number, preserving the waveform symmetries. The proposed strategies operate upto the six-step mode, maintaining the proportionality between the reference magnitude and the fundamental voltage generated throughout. These two strategies lead to lesser harmonic distortion as well as lesser peak current over the conventional space vector strategy (CSVS) in the high speed ranges of constant $V / F$ drives. The reduction in the harmonic distortion over CSVS subject to a given maximum switching frequency $\left(F_{\mathrm{SW}}(\mathrm{MAX})\right)$ of the inverter is demonstrated theoretically as well as experimentally for $F_{\mathrm{SW}(\mathrm{MAX})}=750$ $\mathrm{Hz}$ and $450 \mathrm{~Hz}$, both with and without overmodulation. The best reduction in the distortion is as high as $30 \%$ to $50 \%$ in the different cases considered. Further, these two PWM strategies are also useful in reducing the switching frequency of the inverter over sine-triangle PWM and CSVS subject to an upper limit on the harmonic distortion.
\end{abstract}

Index Terms-Fourier series, harmonic distortion, induction motor drives, inverters, overmodulation, pulse width modulation, space vector, variable speed drives, vectors.

\section{INTRODUCTION}

I $\mathrm{N}$ voltage source inverter fed high-power induction motor drives, due to high losses in the devices, the switching frequency $\left(F_{\mathrm{SW}}\right)$ is quite low [1], and the harmonic distortion is high [2]. The distortion can be reduced by going in for more complex topologies like multilevel inverters [2], split-phase drives [2], [3] etc. Alternatively, it is possible to achieve an improved performance even with a two-level inverter by designing suitable high-performance pulse width modulation (PWM) techniques. Two such open-loop, real-time PWM techniques are developed here.

The harmonic distortion is especially high at higher speeds as the ratio of the switching frequency $\left(F_{\mathrm{SW}}\right)$ to the fundamental frequency $\left(F_{1}\right)$, or the pulse number $\left(P=F_{\mathrm{SW}} / F_{1}\right)$, is low at such speeds. Use of bus-clamping [4]-[11] leads to lesser harmonic distortion at such speeds subject to a given switching frequency [4]-[6], [9] or a given pulse number [10], [11] as the case may be. Lack of synchronization between the PWM wave-

Manuscript received December 8, 2000; revised October 8, 2001. Recommended by Associate Editor G. K. Dubey.

The authors are with the Department of Electrical Engineering, Indian Institute of Science, Bangalore 560 012, India (e-mail: gnar@ieee.org; vtran@ee.iisc.ernet.in).

Publisher Item Identifier S 0885-8993(02)02178-6. form and its fundamental leads to beat phenomenon, the mitigation of which is quite involved [12]. The beat frequencies can be avoided by using synchronized PWM techniques. The space vector approach to PWM [9]-[11] offers certain flexibilities in the design of PWM strategies like double switching of a phase within a subcycle, clamping of two phases within a subcycle etc. [10]. A judicious exploitation of these flexibilities results in improvement in the performance of drives [10], [11]. In this paper, the principles governing the generation of space vector-based synchronized PWM waveforms are reviewed. Two new space vector-based synchronized bus-clamping PWM strategies are proposed.

The total harmonic distortion factor $\left(I_{\mathrm{THD}}\right)$ of the no-load current waveform is a performance measure suitable for PWM strategies for high power drives [2]. An equivalent quantity, which is independent of the motor parameters, is the weighted total harmonic distortion factor ( $\left.V_{\mathrm{WTHD}}\right)$ of the line voltage waveform

$$
I_{\mathrm{THD}}=\frac{\sqrt{\sum I_{n}^{2}}}{I_{1}}, \quad V_{\mathrm{WTHD}}=\frac{\sqrt{\sum\left(V_{n} / n\right)^{2}}}{V_{1}}, \quad n \neq 1
$$

where $I_{1}$ and $I_{n}$ are the RMS values of the fundamental and $n$th harmonic currents respectively, and $V_{1}$ and $V_{n}$ are the RMS values of the fundamental and $n$th harmonic voltages respectively. In the present work, the performances of the PWM strategies are evaluated theoretically in terms of $V_{\text {WTHD }}$, and verified experimentally in terms of $I_{\mathrm{THD}}$.

The modulation index $M$ is defined as the ratio of the fundamental voltage generated to the fundamental voltage corresponding to the six-step operation. A two-level voltage source inverter and the voltage vectors produced by it are shown in Fig. 1(a) and (b), respectively. The modulation index $(M)$ is controlled using the magnitude of the reference voltage $\left(V_{\mathrm{REF}}\right)$. The ideal relationship between $M$ and $V_{\mathrm{REF}}$ is as given in

$$
M=(0.907 / 0.866) * V_{\mathrm{REF}} \text {. }
$$

The proposed strategies can operate upto the six-step mode, i.e., $M=1$, thereby providing the maximum possible DC bus utilization. Also, the modulator gain is maintained constant as per (2) upto $M=1$.

\section{PRINCIPLES}

In space vector approach to PWM, the reference vector is sampled once in every subcycle, $T_{S}$, and an average vector equal 


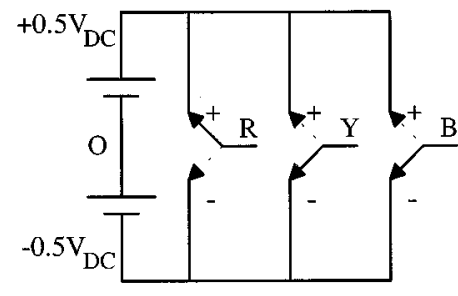

(a)

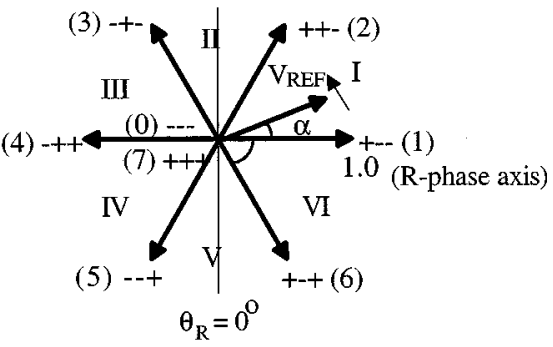

(b)

Fig. 1. (a) Voltage source inverter and (b) voltage vectors produced by it. $\theta_{R}=$ Angle of $R$-phase fundamental voltage. I, II, III, IV, V, and VI: Sectors.

to the sampled reference vector (or "sample," for short) is generated by time-averaging of the different voltage vectors produced by the inverter. For a sample in sector I as shown in Fig. 1(b), the durations for which the active state 1 , the active state 2 , and the two zero states together must be applied are given by $T_{1}, T_{2}$, and $T_{Z}$, respectively, in

$$
\begin{aligned}
T_{1} & =T_{S} * V_{\mathrm{REF}} \sin \left(60^{\circ}-\alpha\right) / \sin \left(60^{\circ}\right) \\
T_{2} & =T_{S} * V_{\mathrm{REF}} \sin (\alpha) / \sin \left(60^{\circ}\right) \\
T_{Z} & =T_{S}-T_{1}-T_{2} .
\end{aligned}
$$

The division of $T_{Z}$ between the two zero states is a degree of freedom in the design of PWM strategies in the space vector domain [5], [6], [9], [10], [13]. In general, an equal division of $T_{Z}$ leads to lesser ripple current [5], [13]. Sequences 0127 and 7210, with such an equal apportioning of $T_{Z}$, are used in conventional space vector modulation to generate a given sample in sector I. Sequences like $\mathbf{0 1 2}, \mathbf{2 1 0}, \mathbf{7 2 1}$, or $\mathbf{1 2 7}$, which use only one zero state, can also be used to generate the same sample. Further, it is also possible to use sequences 0121, 1210, 7212, or 2127 to generate the sample, dividing one of the active state times, $T_{1}$ or $T_{2}$, into two equal intervals [10]. Depending on the choice of the zero state used, either $R$-phase gets clamped to the positive bus or $B$-phase remains clamped to the negative bus. In bus-clamping PWM, the two zero states are used alternately over $60^{\circ}$ durations. The zero state used is usually changed at the middle of every sector for considerations of symmetry as shown in Fig. 2. Use of the zero state 7 from the middle of sector VI to that of sector I leads to $60^{\circ}$ clamping as shown in Fig. 2(a). Use of the zero state $\mathbf{0}$ during the said duration results in $30^{\circ}$ clamping as shown in Fig. 2(b).

The possible inverter states at an arbitrary instant $\theta_{R}=\left(90^{\circ}-\theta\right)$, falling within sector VI, are listed in Col.1 of Table I(a). To maintain half wave symmetry (HWS), 3 -shase symmetry(3PS) and quarter wave symmetry (QWS), the corresponding states at $\theta_{R}=\left(270^{\circ}-\theta\right),\left(210^{\circ}-\theta\right)$, $\left(150^{\circ}-\theta\right)$ and $\left(90^{\circ}+\theta\right)$ must be as shown in columns two through five, respectively, in Table I(a). Putting $\theta=\left(30^{\circ}-\phi\right)$, the last two columns in Table I(a) are reproduced in Table I(b). This table gives the conditions to be satisfied in sector I for the preservation of all the symmetries, and can be generalized to any sector.

To satisfy the conditions for HWS and 3PS, there must be an equal number of samples and at identical positions in every sector. (i.e., the sampling frequency must be an integral multiple of six times the fundamental frequency.) Similar sequences must

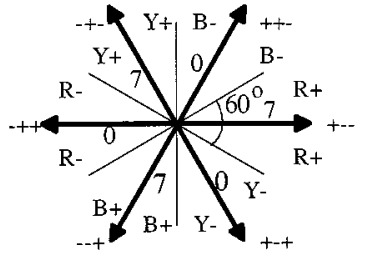

(a)

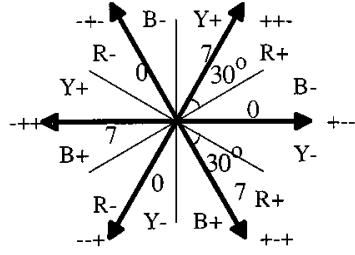

(b)
Fig. 2. Types of bus clamping: (a) $60^{\circ}$ clamping and (b) $30^{\circ}$ clamping.

be used to generate samples at identical locations in different sectors. Thus, in space vector terms, the design of a real-time, synchronized PWM strategy involves the selection of the following three, satisfying the conditions for waveform symmetries and ensuring no simultaneous switching of more than one phase.

1) Number of samples per sector $(N)$.

2) Positions of samples within a sector.

3) Switching sequences used to generate every sample in a sector.

A sample can be generated in sector I with either the $R$-phase clamped to the positive bus or the $B$-phase clamped to the negative bus. Hence, these two are termed as the "clampable phases" of sector I. On the other hand, $Y$-phase must be switched for any transition from one active state to the other, and cannot remain clamped. Hence, this phase is termed as the "unclampable phase" of sector I. Application of Table I(b) to the centre of sector I shows that $Y$-phase must switch at this instant, which corresponds to a zero-crossing of the $Y$-phase fundamental voltage. The state at the starting instant of a sector is termed as "sector initial state." If the sector initial state for sector I is $\mathbf{0}$ or $\mathbf{7}$, then every phase must switch an odd number of times within the sector. If it is the active state 1 , then only $Y$-phase phase must switch an odd number of times, while the two clampable phases must switch an even number of times within the sector. The total number of switchings of the three phases within a sector must be odd. These rules can be generalized to any sector as follows.

1) The unclampable phase must switch at the centre of the sector.

2) The unclampable phase must switch an odd number of times within the sector.

3) The two clampable phases must switch an equal number of times, either odd or even, within the sector.

The above rules are helpful in the construction of sequences as will be demonstrated in the following section. 
TABLE I

CONDITIONS FOR PRESERVATION OF SYNCHRONISATION, HWS, 3PS AND QWS IN SECTOR I

\begin{tabular}{|c|c|c|c|c|c|c|}
\hline $\begin{array}{l}s\left(90^{\circ}-\theta\right), \\
0^{\circ}<\theta<60^{\circ}\end{array}$ & $\begin{array}{l}s\left(270^{\circ}-\theta\right) \\
\text { for } H W S\end{array}$ & $\begin{array}{l}s\left(210^{\circ}-\theta\right) \\
\text { for } 3 P S\end{array}$ & $\begin{array}{l}s\left(150^{\circ}-\theta\right) \text { for } \\
\text { HWS \& 3PS }\end{array}$ & $\begin{array}{l}\mathrm{s}\left(90^{\circ}+\theta\right) \text { for } \\
\text { QWS \& 3PS }\end{array}$ & $\begin{array}{l}S\left(120^{\circ}-\phi\right), \\
0^{\circ} \leq \phi \leq 30^{\circ}\end{array}$ & $\begin{array}{c}\mathrm{s}\left(120^{\circ}+\phi\right) \text { for } Q W S \\
\text { HWS \& } 3 \text { PS }\end{array}$ \\
\hline --- & +++ & --- & +++ & --- & $-m$ & +++ \\
\hline+++ & --- & +++ & --- & +++ & +++ & --- \\
\hline+- & -++ & -+- & ++- & +-- & +-- & ++ \\
\hline+-+ & -+- & ++- & +-- & ++- & ++ & +-- \\
\hline
\end{tabular}

TABLE II

BASIC Bus Clamping STRATEGY-II (BBCS-II)

\begin{tabular}{c|c|c|c|c|c|c|c}
\hline $\begin{array}{c}\text { Sector } \\
\text { initial } \\
\text { state }\end{array}$ & $\begin{array}{c}\text { Sequences for the } \\
\text { first (N-2)/2 } \\
\text { samples }\end{array}$ & $\begin{array}{c}\text { Sequences for } \\
\text { the middle } \\
\text { two samples }\end{array}$ & $\begin{array}{c}\text { Sequences for the } \\
\text { last (N-2)/2 } \\
\text { samples }\end{array}$ & $\begin{array}{c}\text { Sector } \\
\text { final } \\
\text { state }\end{array}$ & (N-2)/2 & N & $\begin{array}{c}\text { Type of } \\
\text { clamping }\end{array}$ \\
\hline 0 & $012,210, . .210$ & 012,127 & $721,127, . .127$ & 7 & $2 n-2$ & $4 n-2$ & $30^{\circ}$ \\
1 & $127,721, . .127$ & 721,210 & $012,210, . .012$ & 2 & $2 n-1$ & $4 n$ & $60^{\circ}$ \\
7 & $721,127, \ldots 127$ & 721,210 & $012,210, \ldots 210$ & 0 & $2 n-2$ & $4 n-2$ & $60^{\circ}$ \\
\hline
\end{tabular}

$\mathrm{N}$ : number of samples per sector, $\mathbf{n}$ : any positive integer

\section{PRoposed PWM StRATEGIES}

Three space vector-based bus-clamping PWM strategies have been reported recently-asymmetric zero-changing strategy (AZCS), basic bus clamping strategy and boundary sampling strategy [10], [11]. The last two are designated as basic bus clamping strategy-I (BBCS-I) and boundary sampling strategy-I (BSS-I), respectively, in the present work. Conventional space vector strategy (CSVS) produces PWM waveforms with $P=3,9,15,21 \ldots$; BBCS-I with $P=7,11,15,19 \ldots$; BSS-I and AZCS with $P=5,9,13,17 \ldots$. Two new bus-clamping PWM strategies, namely basic bus clamping strategy-II (BBCS-II) and boundary sampling strategy-II (BSS-II), are developed here. BBCS-II produces PWM waveforms preserving all the symmetries with $P=5,9,13,17 \ldots$, and BSS-II with $P=7,11,15,19 \ldots$

\section{A. Basic Bus Clamping Strategy-II (BBCS-II)}

The objective here is to develop a bus-clamping PWM strategy which can produce PWM waveforms with $P=5,9,13,17 \ldots$, preserving the waveform symmetries. The total number of switchings of the three phases or the number of state transitions within a sector is equal to the pulse number $P$. To generate an arbitrary sample, at least one switching of the unclampable phase and one switching of one of the clampable phases is needed. Hence, given $N$ samples per sector, at least $2 N$ switchings are required. However, under this condition, the zero state used cannot be changed. To change the zero state used once in every sector at least one extra switching is needed. Therefore, $P=(2 N+1)$ is the minimum value of $P$ required to generate $N$ samples per sector, maintaining the waveform symmetries [10]. If $P=5,9,13,17 \ldots$ and $P=2 N+1$, then $N=2,4,6,8 \ldots$ Thus, $N$ must be even for this strategy.

Since $N$ is even, the unclampable phase can be made to switch $(N+1)$ times to maintain the symmetries, making the extra switching pertain to this phase. The two clampable phases must switch $(N / 2)$ times each. The unclampable phase must switch at the instant corresponding to the centre of the sector. To ensure this, one option is to have a sample at the centre of the sector, i.e., at $\alpha=30^{\circ}$, and use the sequence 0127 or 7210 to generate it. However, the extra switching does not pertain to the unclampable phase as required. Alternatively, there can be two samples at the middle of the sector, equidistant from $\alpha=30^{\circ}$ and on either side of it, which use the sequences $\mathbf{0 1 2}, \mathbf{1 2 7}$ or $\mathbf{7 2 1}, \mathbf{2 1 0}$. This meets the requirement as the extra switching pertains to the unclampable phase in this case. Hence this is chosen. Thus, $N$ is even, and the samples are positioned symmetrically about the centre of the sector with no sample at $\alpha=30^{\circ}$ in this strategy.

For construction of sequences, the samples within a sector can be grouped into the first $(N-2) / 2$ samples, the middle two samples and the last $(N-2) / 2$ samples as shown in Table II. If $\mathbf{0}$ be the sector initial state, then the sequences $012,210, \ldots, 012,210$ must be used for the first $(N-2) / 2$ samples; sequences $\mathbf{0 1 2 , 1 2 7}$ must be used for the middle two samples to change the zero state used from $\mathbf{0}$ to 7 ; and sequences $\mathbf{7 2 1}, \mathbf{1 2 7}, \ldots, \mathbf{7 2 1}, \mathbf{1 2 7}$ for the last $(N-2) / 2$ samples as shown in Table II. Sequences are constructed in a similar fashion for the other two sector initial states.

In case of sector initial states $\mathbf{0}$ and $\mathbf{7}$, the sequences for the first $(N-2) / 2$ samples start and end with the same state, implying that $(N-2) / 2$ must be even, or $(N-2) / 2=(2 n-$ 2 ), where $n$ is any positive integer. Hence, $N=4 n-2=$ $2,6,10 \ldots$ as shown in Table II. Therefore, $P=2 N+1=$ $5,13,21 \ldots$ In case of sector initial state $1,(N-2) / 2$ must be odd, or $(N-2) / 2=(2 n-1)$, where $n$ is any positive integer. Hence $N=4 n=4,8,12 \ldots$, and $P=2 N+1=9,17,25 \ldots$.

In case of sector initial states $\mathbf{1}$ and $\mathbf{7}$, the zero state $\mathbf{7}$ alone is used between the middle of sector VI and that of sector I. For sector initial state $\mathbf{0}$, the zero state $\mathbf{0}$ is used in the said duration. The former leads to $60^{\circ}$ clamping, and the latter results in $30^{\circ}$ clamping as shown in Fig. 2. 
TABLE III

BOUNDARY SAMPLING STRATEGY-II (BSS-II)

\begin{tabular}{c|c|c|c|c|c|c}
\hline $\begin{array}{c}\text { Sequence for } \\
\text { the sample at } \\
\alpha=0^{\circ}\end{array}$ & $\begin{array}{c}\text { Sequences for the } \\
\text { first }(\mathrm{N}-3) / 2 \\
\text { samples }\end{array}$ & $\begin{array}{c}\text { Sequence for } \\
\text { the middle two } \\
\text { samples }\end{array}$ & $\begin{array}{c}\text { Sequences for the } \\
\text { last }(\mathrm{N}-3) / 2 \\
\text { samples }\end{array}$ & (N-3)/2 & $\mathrm{N}$ & $\begin{array}{c}\text { Type of } \\
\text { clamping }\end{array}$ \\
\hline 010 & $012,210, . .210$ & 012,127 & $721,127, .127$ & $2 \mathrm{n}-2$ & $4 \mathrm{n}-1$ & $30^{\circ}$ \\
101 & $127,721, . .127$ & 721,210 & $012,210, . .210$ & $2 \mathrm{n}-1$ & $4 \mathrm{n}+1$ & $60^{\circ} *$ \\
\hline \multicolumn{6}{c}{$\mathrm{N}:$ number of samples per sector, $\mathrm{n}:$ any positive integer } \\
* with a small discontinuity at the centre of the clamping duration
\end{tabular}

(a)

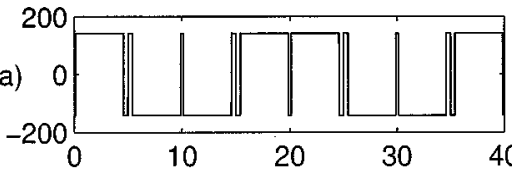

(c)

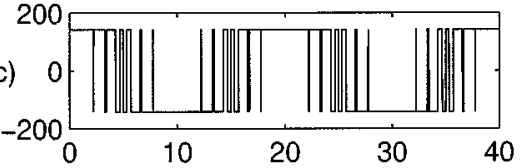

(e)

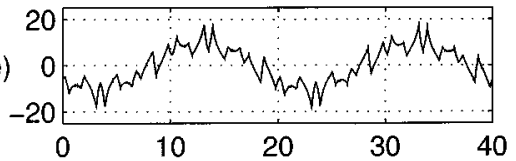

(g)

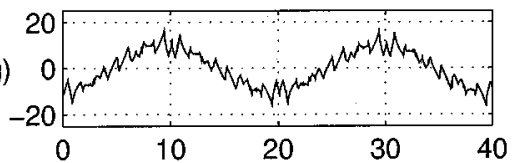

(i)

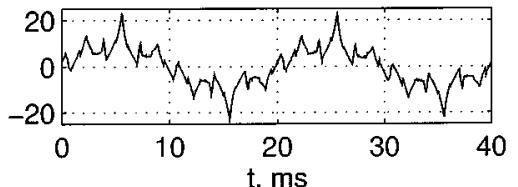

(b)

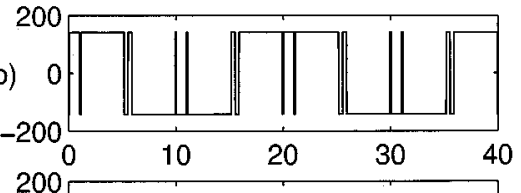

(d)

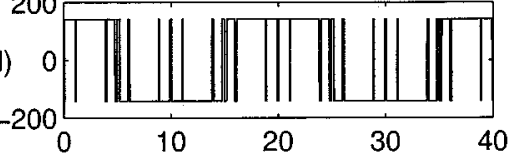

(f)

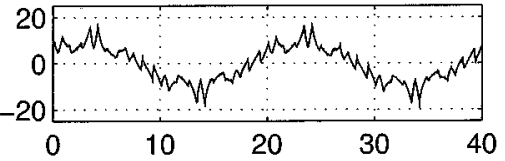

(h)

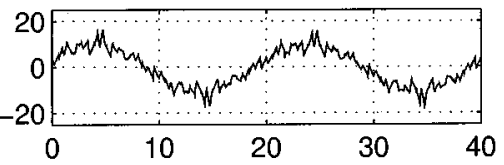

(j)

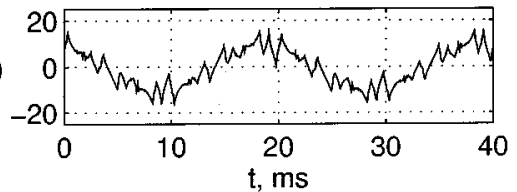

Fig. 3. (a)-(d) Typical pole voltage waveforms and (e)-(j) experimental no-load current waveforms at $M=0.907$ and $F_{1}=50 \mathrm{~Hz}$. (a), (e): BBCS-II, $N=$ $2,30^{\circ}$ clamping $(P=5)$; (b), (f) BSS-II, $N=3(P=7)$; (c), (g) BBCS-II, $N=6,60^{\circ}$ clamping $(P=13)$; (d), (h) BBCS-II, $N=6,30^{\circ}$ clamping $(P=13)$; (i) CSVS, $(N=3)(P=9)$; (j) AZCS, $(N=4)(P=9)$. Measured values of $I_{\mathrm{THD}}$ are (e) 0.3526, (f) 0.3061, (g) 0.2647, (h) 0.2464, (i) 0.4858, and (j) 0.3369 .

Thus, this strategy generates PWM waveforms, preserving all the symmetries, with $P=5,9,13,17 \ldots$ It generates two types of PWM waveforms, namely the $60^{\circ}$ clamping type and the $30^{\circ}$ clamping type. With $P=5,13,21 \ldots$, it produces both the types of PWM waveforms. However, with $P=9,17,25 \ldots$, it generates only the $60^{\circ}$ clamping type.

\section{B. Boundary Sampling Strategy-II (BSS-II)}

To generate a sample on a sector boundary, only one active vector is needed. A sample on the boundary between Sectors VI and I can be generated using the sequence $\mathbf{0 1 0}$ or 101, with either $T_{0}$ or $T_{1}$ divided into two equal halves. Such a sample can be termed as "boundary sample," and the sequence as "boundary sequence" [10]. In boundary sampling strategy-II, there is a sample on every sector boundary, and the zero-changing is done as in BBCS-II. The samples in a sector can be grouped as the boundary sample, the middle two samples, the $(N-3) / 2$ samples before and after the middle two samples as shown in Table III. The sequences can be constructed considering the two possible boundary sequences, namely 010 or 101.

If the boundary sequence is $\mathbf{0 1 0}$, then the sequences 012,210 are used for generating the alternate samples in the next $(N-3) / 2$ samples as shown in Table III. Then, zero-changing is done over the middle two samples using the sequences 012,127 . Sequences 721,127 are used to generate alternate samples in the $(N-3) / 2$ samples that follow the middle two samples. In this case $(N-3) / 2$ must be even, i.e., $(N-3) / 2=(2 n-2)$, where $n$ is any positive integer. Hence $N=(4 n-1)=3,7,11 \ldots$ and $P=7,15,23 \ldots$ The choice of the zero state leads to $30^{\circ}$ clamping.

If the boundary sequence used is $\mathbf{1 0 1}$, the sequences must be as shown in Table III. In this case it can be seen that $(N-3) / 2$ must be odd, i.e., $N=(4 n+1)$, where $n$ is any positive integer. Hence $N=5,9,13 \ldots$ and $P=11,19,27 \ldots$ The sequences used lead to $60^{\circ}$ clamping with a small discontinuity at the centre of the clamping duration. This strategy produces PWM waveforms with all the symmetries with $P=7,11,15,19 \ldots$

\section{Waveforms and Spectra}

The PWM strategies are implemented on an INTEL 80C196KB microcontroller. The experimental prototype is a $200 \mathrm{~V}, 3 \mathrm{~kW}, 50 \mathrm{~Hz}$ induction motor fed from a $5 \mathrm{kVA}$ IGBT inverter. The pole voltage waveforms corresponding to $M=0.907$ are presented for select cases in Fig. 3(a)-(d). The experimental no-load current waveforms, corresponding 

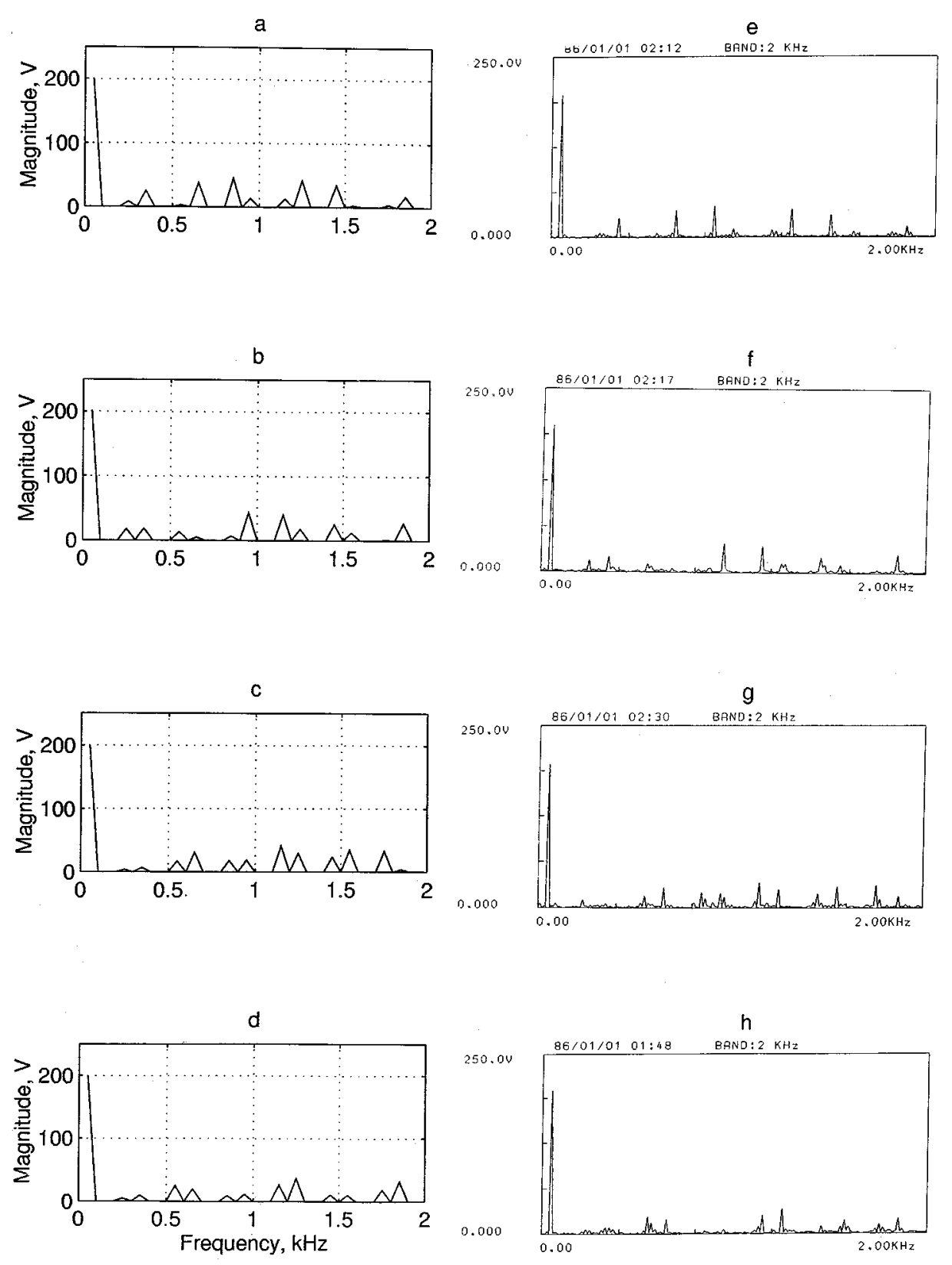

Fig. 4. (a)-(d) Theoretical spectra and (e)-(h) measured spectra of line voltage waveforms corresponding to Fig. 3(a)-(d). $V_{\text {WTHD }}$ values are (a) 0.0296 , (b) 0.0283 , (c) 0.0225 , and (d) 0.0209 .

to these pole voltages, are shown in Fig. 3(e)-(h), respectively. The ripple currents produced by the proposed strategies are characterized by double peaks due to the switching sequences used in the middle two subcycles in every sector. The double peaks of the ripple current add to the fundamental current in case of $30^{\circ}$ clamping. They oppose the fundamental current in case of $60^{\circ}$ clamping. The no-load current waveforms corresponding to CSVS and AZCS [10], [11], both with $P=9$, are presented in Fig. 3(i) and (j), respectively, for comparison. It can be observed that both BBCS-II with $P=5$ and BSS-II with $P=7$ lead to lesser $I_{\text {THD }}$ as well as peak current than CSVS with $P=9$. BSS-II with $P=7$ leads to lesser $I_{\text {THD }}$ than even AZCS with $P=9$ or any other real-time synchronized PWM strategy with a uniform sampling rate with $P=9$ [7]-[11], [14].
The theoretical line voltage spectra, corresponding to the pole voltage waveforms in Fig. 3(a)-(d), are shown in Fig. 4(a)-(d), respectively. The corresponding measured spectra are presented in Fig. 4(e)-(h), respectively. The theoretical and measured spectra agree well. It can be seen from Figs. 3 and 4 that the harmonic distortion is less in case of $30^{\circ}$ clamping than with $60^{\circ}$ clamping. This is in line with the earlier observations at low as well as high switching frequencies [4]-[6], [9]-[11].

\section{OVERMODULATION}

The whole range of modulation upto the six-step mode $(M=1)$ can be divided into three zones, namely circular modulation zone, overmodulation zone-I and overmodulation zone-II, as shown in Fig. 5. Before PWM calculations, the 


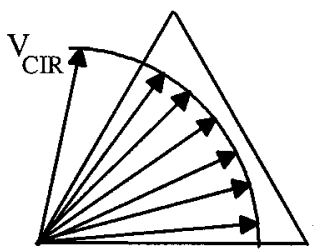

(a)

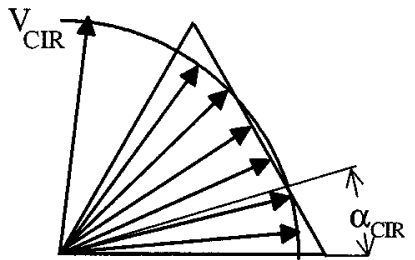

(b)

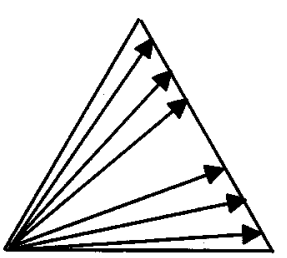

(c)

Fig. 5. Different zones of modulation: (a) circular modulation zone, (b) overmodulation zone-I, and (c) overmodulation zone-II.

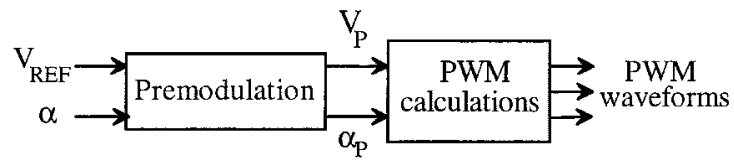

Fig. 6. Premodulation for overmodulation and linearity.

sample of the reference vector must be suitably corrected or "premodulated" in every subcycle during overmodulation [15]-[17], and also to maintain the modulator gain constant in the whole range of modulation [16] as shown in Fig. 6. The premodulation procedure adopted for the two proposed strategies is explained in this section.

In overmodulation zone-I, $M$ is increased by increasing the radius of the circular trajectory $V_{\mathrm{CIR}}$ as in the circular modulation zone. This zone starts from $V_{\mathrm{CIR}}=0.866 / \cos \left(\omega T_{S} / 2\right)$, when the tips of the middle two average vectors in every sector touch the hexagon, connecting the tips of the six active vectors of the inverter. This zone extends upto $V_{\mathrm{CIR}}=0.866 / \cos \left(30^{\circ}-\omega T_{S} / 2\right)$ for BBCS-II and upto $V_{\mathrm{CIR}}=1$ for BSS-II, when the tips of all the average vectors touch the hexagon. The starting and the ending of this zone mean the same for BBCS-II with $N=2$. The variation in $M$ with $V_{\mathrm{CIR}}$ in the zone-I for BBCS-II with $N=4,6$ and for BSS-II with $N=3,5,7$ is shown in Fig. 7(a) and (b), respectively. As every additional pair of average vectors saturates with increase in $V_{\mathrm{CIR}}$, the slope of a given $M$ versus $V_{\mathrm{CIR}}$ curve decreases, making it piecewise linear. Using $M$ versus $V_{\mathrm{CIR}}$ and (2), the relationship $V_{\mathrm{CIR}}$ versus $V_{\mathrm{REF}}$ is arrived at. The $V_{\mathrm{CIR}}$ versus $V_{\mathrm{REF}}$ curves for BBCS-II and BSS-II are shown in Fig. 7(c) and (d), respectively. During premodulation in zone-I, appropriate value of $V_{\mathrm{CIR}}$ is chosen for a given $V_{\mathrm{REF}}$ using these curves. The magnitude $\left(V_{P}\right)$ and angle $\left(\alpha_{P}\right)$ of the premodulated sample are then calculated as given by

$$
\begin{aligned}
V_{P(\mathrm{MAX})} & =0.866 / \cos \left(30^{\circ}-\alpha\right) \\
V_{P} & =V_{\mathrm{CIR}}, \quad \text { if } V_{\mathrm{CIR}} \leq V_{P(\mathrm{MAX})} \\
& =V_{P(\mathrm{MAX})}, \quad \text { if } V_{\mathrm{CIR}}>V_{P(\mathrm{MAX})} \\
\alpha_{P} & =\alpha .
\end{aligned}
$$

In overmodulation zone-II, the modulation index $M$ is increased by shifting the average vectors generated over different subcycles closer to the nearest sector boundary with their tips touching the hexagon. This is done using a fraction termed as 'angle correction factor' $K_{\mathrm{ANG}}$ as shown in

$$
\begin{aligned}
\alpha_{P} & =K_{\mathrm{ANG}} * \alpha, \quad 0^{\circ} \leq \alpha<30^{\circ} \\
& =30^{\circ}, \quad \alpha=30^{\circ} \\
& =60^{\circ}-K_{\mathrm{ANG}} *\left(60^{\circ}-\alpha\right), \quad 30^{\circ}<\alpha<60^{\circ} \\
V_{P} & =0.866 / \cos \left(30^{\circ}-\alpha_{P}\right) .
\end{aligned}
$$

$M$ is increased upto 1 in this zone by decreasing $K_{\mathrm{ANG}}$ from 1 to 0 . The variation in $M$ with $K_{\mathrm{ANG}}$ for BBCS-II with $N=$ 2, 4, 6 and for BSS-II with $N=3,5,7$ are shown in Fig. 7(e) and (f) respectively. For simplicity, these curves can be approximated by the dotted straight lines shown in Fig. 7(e) and (f). Using the approximate $M$ versus $K_{\mathrm{ANG}}$ curves and (2), the corresponding $K_{\mathrm{ANG}}$ versus $V_{\mathrm{REF}}$ curves are obtained. These are shown in Fig. 7(g) and (h) for BBCS-II and BSS-II, respectively. During premodulation in zone-II, the appropriate value of $K_{\mathrm{ANG}}$ for the given $V_{\mathrm{REF}}$ is chosen from these curves. $\alpha_{P}$ and $V_{P}$ are then calculated as per (5).

With very low pulse numbers of 5 and 7 as in the case of BBCS-II with $N=2$ and BSS-II with $N=3$, the relationship between $M$ and $V_{\text {CIR }}$ is not linear even in the circular modulation zone. Hence the term "circular modulation zone" is used here instead of "linear modulation zone." Here again the proportionality is maintained by suitable premodulation.

The experimental no-load current waveforms corresponding to BBCS-II, BSS-II and CSVS with nominal pulse numbers of 5, 7, and 9, respectively, are presented in Fig. 8. The waveforms are shown for $M=0.92,0.96$ and 1.0, demonstrating the transition from PWM to six-step. It can be seen that BBCS-II and BSS-II perform better than CSVS over this whole range excluding the six-step mode, which is common for all the strategies. The performance is superior both in terms of $I_{\text {THD }}$ as well as peak current.

\section{Performance Evaluation}

The linearity between the reference magnitude and the modulation index is verified experimentally for BBCS-II with $N=2$ and $30^{\circ}$ clamping, and for BSS-II with $N=3$. The results for the two cases are shown in Fig. 9(a). The performance of the two proposed strategies are evaluated over the whole range of modulation upto the six-step mode. The $V_{\mathrm{WTHD}}$ versus $M$ characteristics of BBCS-II and BSS-II are shown in Fig. 9(b) and (c), respectively, for select values of nominal pulse number $\left(P_{\mathrm{NOM}}\right)$, which is the pulse number prior to the onset of pulse dropping. 


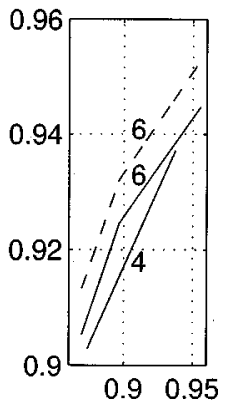

(a)

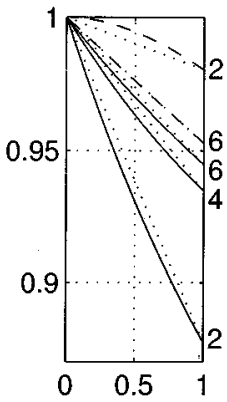

(e)

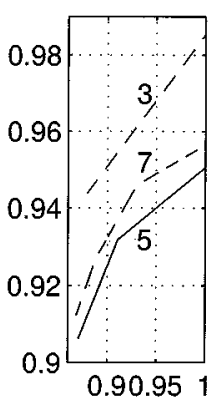

(b)

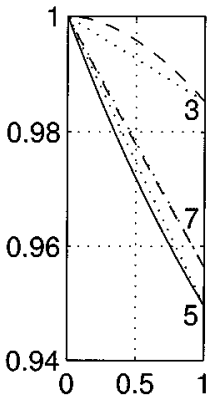

(f)

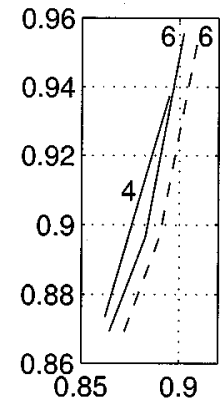

(c)

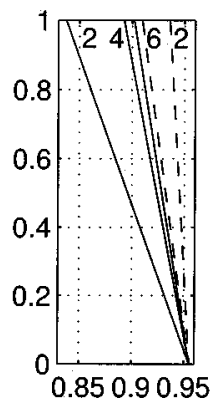

$(\mathrm{g})$

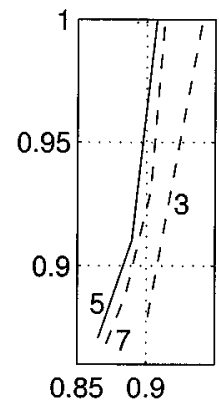

(d)

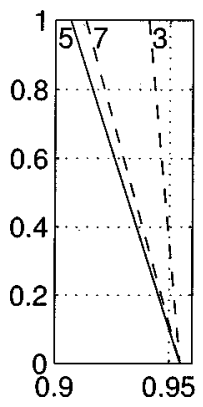

(h)

Fig. 7. Voltage control during overmodulation. (a)-(d) Overmodulation zone-I; (e)-(h) overmodulation zone-II. (a) $M$ versus $V_{C I R}$ for BBCS-II (b) $M$ versus $V_{\text {CIR }}$ for BSS-II; (c) $V_{\text {CIR }}$ versus $V_{\text {REF }}$ for BBCS-II; (d) $V_{\text {CIR }}$ versus $V_{\text {REF }}$ for BSS-II; (e) $M$ versus $K_{\text {AvG }}$ for BBCS-II (f): $M$ versus $K_{\mathrm{ANG}}$ for BSS-II; (g) $K_{\mathrm{ANG}}$ versus $V_{\mathrm{REF}}$ for BBCS-II; (h) $K_{\mathrm{ANG}}$ versus $V_{\mathrm{REF}}$ for BSS-II Solid line: $60^{\circ}$ clamping, dashed line: $30^{\circ}$ clamping, dotted line: approximation.

(a)

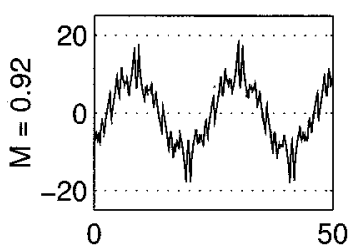

(b)

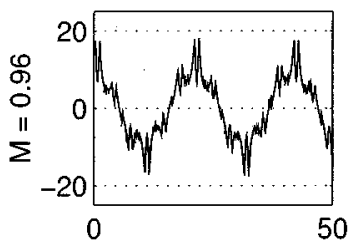

(c)

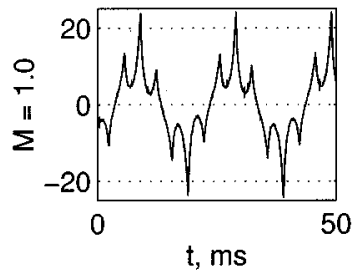

(d)

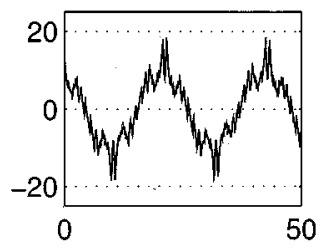

(e)

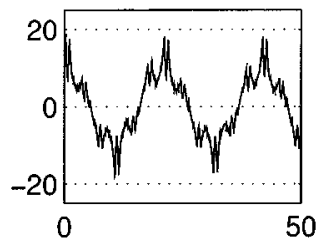

(f)

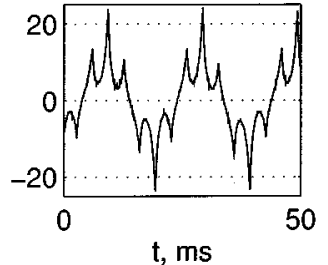

(g)

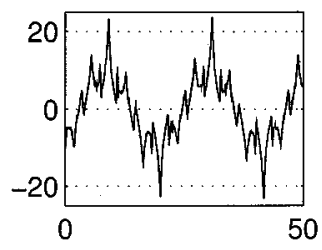

(h)

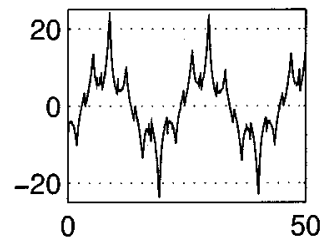

(i)

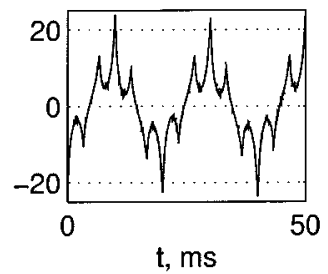

Fig. 8. Experimental no-load current waveforms demonstrating transition from PWM to six-step operation. (a)-(c) BBCS-II, $N=2,30^{\circ}$ clamping $\left(P_{\mathrm{vOM}}=\right.$ 5 ); (d)-(f) BSS-II, $N=3$ ( $P_{\mathrm{NOM}}=7$ ); (g)-(i) CSVS, $N=3$, ( $\left.P_{\mathrm{NOM}}=9\right)$. Measured values of $I_{\mathrm{THD}}$ are (a) 0.3522 , (b) 0.3078 , (c) 0.5385 , (d) 0.3203, (e) 0.3172 , (f) 0.5397, (g) 0.4937 , (h) 0.4942 , and (i) 0.5419 .

Comparison of the $V_{\text {WTHD }}$ versus $M$ characteristics of these two strategies against those of CSVS with $P_{\mathrm{NOM}}=9,15$ and 21 is presented in Fig. 9(d), (e), and (f), respectively. It can be seen that the proposed strategies perform better than CSVS at higher $M$ with the same pulse number or even with lower pulse number. Notable are the superior performances of BBCS-II with
$P_{\mathrm{NOM}}=5$ and BSS-II with $P_{\mathrm{NOM}}=7$ over CSVS with $P_{\mathrm{NOM}}=9$ in the ranges $M>0.84$ and $M>0.72$, respectively, as shown in Fig. 9(d) [see also Fig. 3(e), (f), and (i)]. Thus, there is an improvement in the harmonic distortion along with a considerable reduction in the switching frequency and switching losses in such cases. 
(a)

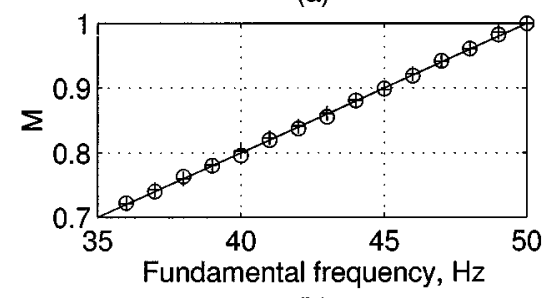

(b)

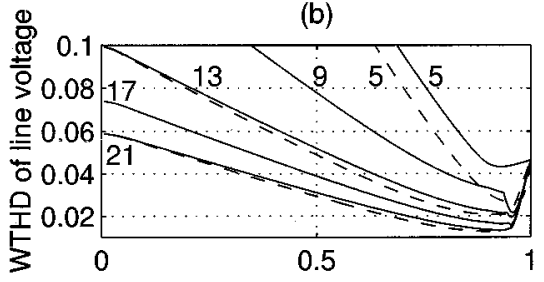

(c)

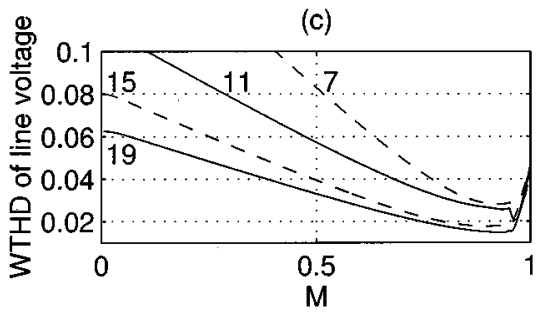

(d)

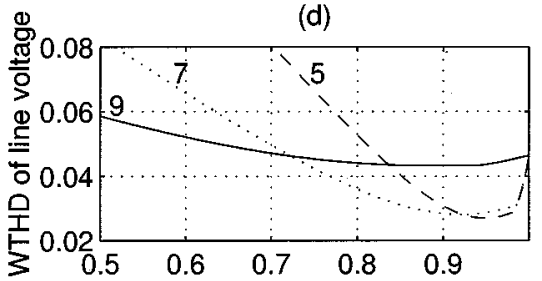

(e)

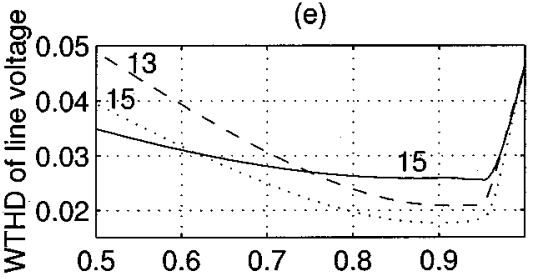

(f)

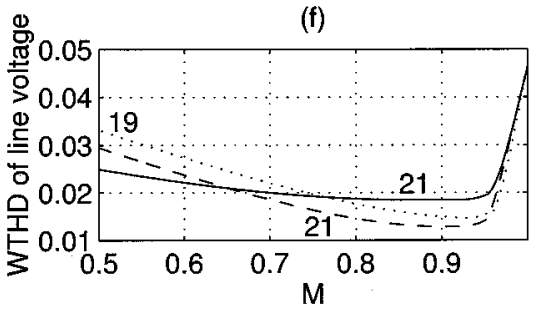

Fig. 9. Performance assessment of the proposed PWM strategies. (a) Experimental verification of linearity of BBCS-II and BSS-II. (b) $V_{\text {WTHD }}$ versus $M$ characteristics of BBCS-II. (c) $V_{\mathrm{WTHD}}$ versus $M$ characteristics of BSS-II. (d)-(f) Comparison of $V_{\mathrm{WTHD}}$ characteristics of BBCS-II and BSS-II against those of CSVS with $P_{\mathrm{NOM}}=9,15$, and 21 , respectively. (a) Solid line: ideal relationship; o: measured value for BBCS-II, $N=2,30^{\circ}$ clamping; $+:$ measured value for BSS-II, $N=3$. (b)-(c): Solid line: $60^{\circ}$ clamping, dashed line: $30^{\circ}$ clamping. (d)-(f): Solid line: CSVS, dashed line: BBCS-II, dotted line: BSS-II.

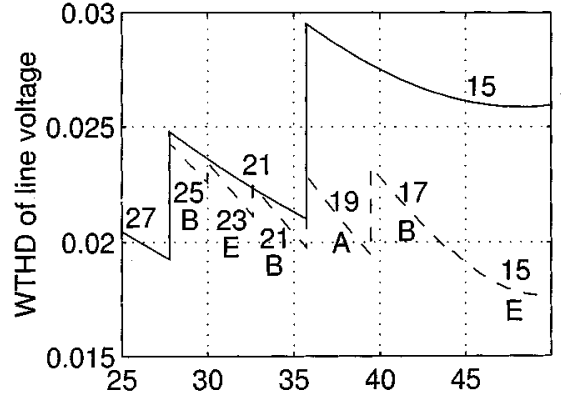

(a)

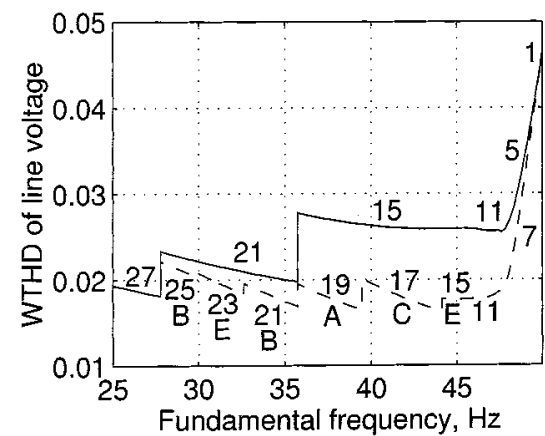

(c)

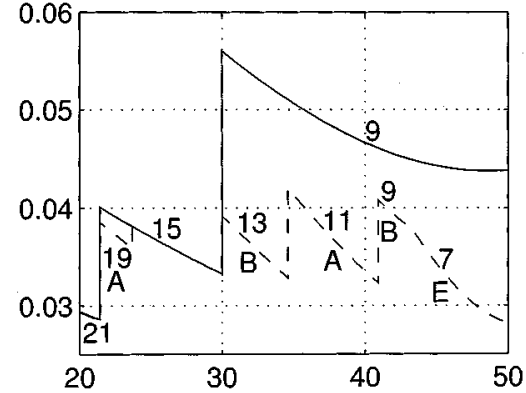

(b)

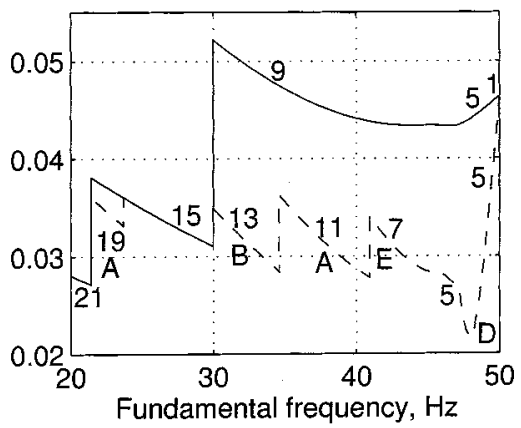

(d)

Fig. 10. Reduction in $V_{\mathrm{WTHD}}$ of constant $V / F$ drive subject to upper limit on switching frequency. (a) $F_{S W(\mathrm{MAX})}=750 \mathrm{~Hz}, M_{\mathrm{MAX}}=0.907$, (b) $F_{\mathrm{SW}(\mathrm{MAX})}=450 \mathrm{~Hz}, M_{\mathrm{MAX}}=0.907$, (c) $F_{\mathrm{SW}(\mathrm{MAX})}=750 \mathrm{~Hz}, M_{\mathrm{MAX}}=1.0$, and (d) $F_{\mathrm{SW}(\mathrm{MAX})}=450 \mathrm{~Hz}, M_{\mathrm{MAX}}=1.0 \mathrm{Solid}$ line: CSVS, dashed line: hybrid PWM. A: BBCS-I, B: BSS-I, C: AZCS, D: BBCS-II, E: BSS-II.

\section{Application to Constant $V / F$ Drives}

A suitable combination of different PWM strategies over different speed ranges can be worked out for reduction in harmonic distortion subject to any given maximum switching frequency $\left(F_{\mathrm{SW}}(\mathrm{MAX})\right)$ of the inverter. Fig. 10(a) and (b) show the $V_{\text {WTHD }}$ versus $F_{1}$ characteristics of a constant $V / F$ drive with $F_{\mathrm{SW}}(\mathrm{MAX})$ of $750 \mathrm{~Hz}$ and $450 \mathrm{~Hz}$, respectively. The max- 


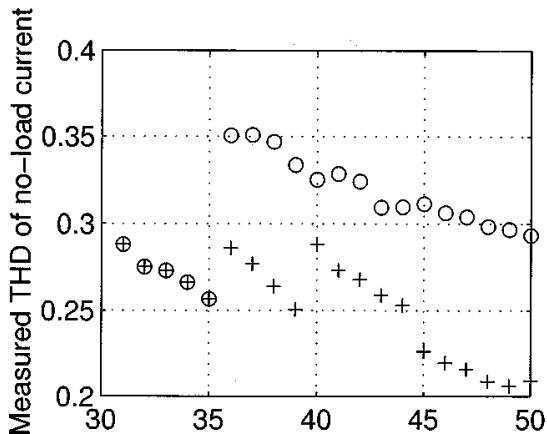

(a)

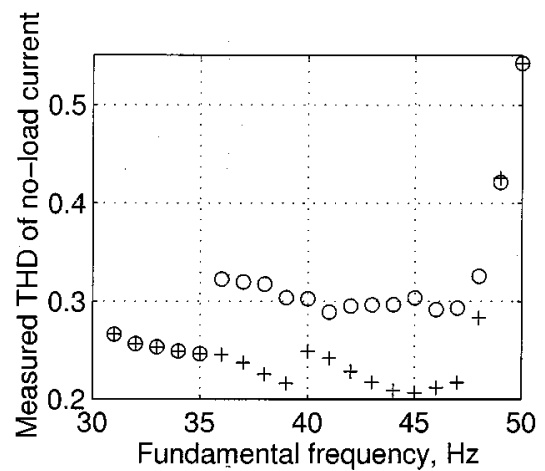

(c)

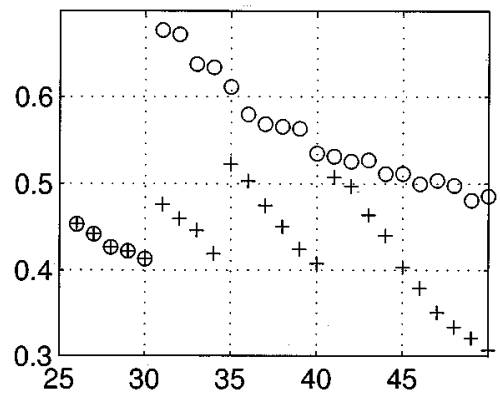

(b)

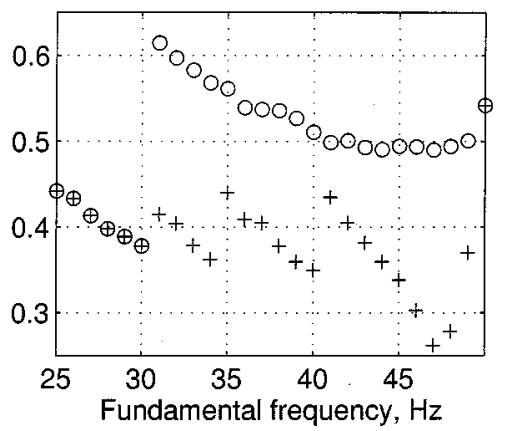

(d)

Fig. 11. Measured $I_{\mathrm{THD}}$ of no-load current waveform of constant $V / F$ drive showing reduction due to hybrid PWM. (a) $F_{\mathrm{SW}(\mathrm{MAX})}=750 \mathrm{~Hz}, M_{\mathrm{MAX}}=$ 0.907 , (b) $F_{\mathrm{SW}(\mathrm{MAX})}=450 \mathrm{~Hz}, M_{\mathrm{MAX}}=0.907$, (c) $F_{\mathrm{SW}(\mathrm{MAX})}=750 \mathrm{~Hz}, M_{\mathrm{MAX}}=1.0$, and (d) $F_{\mathrm{SW}(\mathrm{MAX})}=450 \mathrm{~Hz}, M_{\mathrm{MAX}}=1.0 \mathrm{o}: \mathrm{CSVS},+$ : hybrid PWM.

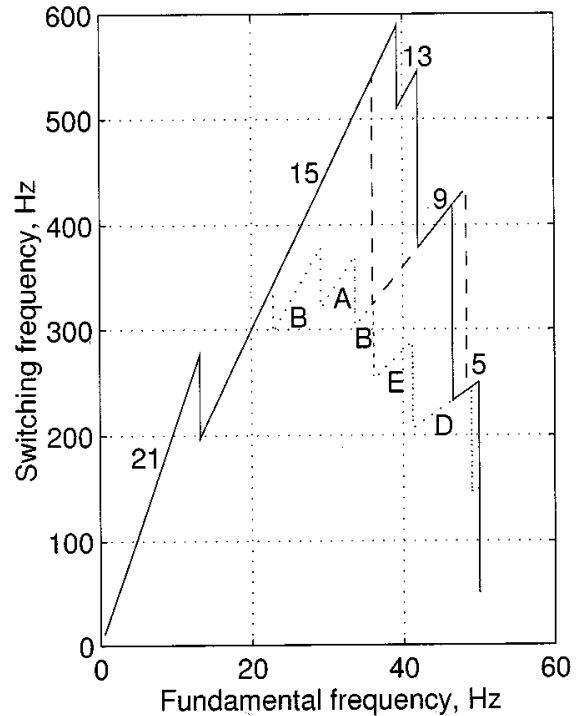

Fig. 12. Reduction in switching frequency due to hybrid PWM subject to $V_{\mathrm{WTHD}(\mathrm{MAX})}=V_{\mathrm{WTHD}(6-\mathrm{STEP})}$. Solid line: sine-triangle PWM, dashed line: CSVS, dotted line: hybrid PWM. A: BBCS-I, B: BSS-I, C: AZCS, D: BBCS-II, E: BSS-II.

imum modulation index $M_{\mathrm{MAX}}=0.907$, and DC bus voltage $V_{\mathrm{DC}}=1.41 * V_{\mathrm{RATED}}$, where $V_{\mathrm{RATED}}$ is the rated line-line voltage of the motor. With only CSVS used, the characteristics are as shown in solid lines. When a combination of CSVS, BBCS-I, BSS-I, AZCS, BBCS-II, and BSS-II is used, the characteristics are as shown in dashed lines. It can be seen that such a hybrid PWM results in a significant reduction of $V_{\text {WTHD }}$ at high speed ranges. The maximum reduction is about $31 \%$ and the average reduction in the frequency range $35.7 \mathrm{~Hz}-50 \mathrm{~Hz}$ is about $25 \%$ for $F_{\mathrm{SW}(\mathrm{MAX})}=750 \mathrm{~Hz}$. The maximum reduction is about $36 \%$ and the average reduction in the range $30 \mathrm{~Hz}-50$ $\mathrm{Hz}$ is about $23 \%$ for $F_{\mathrm{SW}(\mathrm{MAX})}=450 \mathrm{~Hz}$. Fig. $10(\mathrm{c})$ and (d) show the characteristics of a drive with $M_{\mathrm{MAX}}=1.0$ and $V_{\mathrm{DC}}=1.28 * V_{\mathrm{RATED}}$ for $F_{\mathrm{SW}(\mathrm{MAX})}=750 \mathrm{~Hz}$ and $450 \mathrm{~Hz}$, respectively. Here again, a suitable combination of PWM strategies reduces the harmonic distortion considerably. The reduction is verified experimentally on the prototype mentioned in Section III-C. The measured $I_{\text {THD }}$ values, corresponding to Fig. 10(a)-(d), are shown in Fig. 11(a)-(d), respectively.

Another approach to combine PWM strategies is to minimize the switching frequency such that the harmonic distortion at any speed is less than or equal to that at the six-step mode [2]. With $V_{\text {WTHD }} \leq V_{\text {WTHD(6-STEP) }}$, the switching frequency of the inverter over the whole speed range of the drive is shown in Fig. 12. With synchronized sine-triangle PWM, the switching frequency varies as shown in solid lines. With CSVS, the switching frequency is less at medium and higher speeds as shown in dashed lines. With hybrid PWM, the switching frequency is still lesser as shown in dotted lines. The maximum switching frequencies of the inverter in case of synchronized sine-triangle PWM, CSVS and hybrid PWM are $580 \mathrm{~Hz}, 540$ $\mathrm{Hz}$, and $380 \mathrm{~Hz}$, respectively. Thus, a combination of PWM strategies leads to lesser switching frequencies, and thereby to lesser switching losses.

In all the above cases illustrated in Figs. 10-12, it can be seen that the two proposed strategies are the ones used at the highest 
speed range. This demonstrates the superiority in the performance of these two strategies at this range over other comparable strategies.

\section{CONCLUSION}

Two new synchronized, bus-clamping PWM strategies based on the space vector approach have been proposed. The two strategies together can generate PWM waveforms with any odd pulse number, maintaining half wave symmetry, quarter wave symmetry, and three-phase symmetry. The ripple current produced by these strategies is characterized by double peaks due to the switching sequences used in the middle two subcycles of every sector. This results in lesser peak current than CSVS and many other comparable strategies at higher speeds. The proposed strategies can be employed gainfully either to reduce the harmonic distortion subject to an upper limit on the switching frequency, or to reduce the switching frequency, and hence the switching losses, subject to an upper limit on the harmonic distortion in constant $V / F$ drives. At speeds close to the base speed, these strategies perform better than other comparable open-loop, real-time synchronized PWM strategies with a uniform sampling rate. The proposed strategies are useful in high power drives where the inverters switch at low frequencies and the machines have low leakage inductance.

\section{REFERENCES}

[1] S. Bernet, R. Teichmann, A. Zuckerberger, and P. K. Steimer, "Comparison of high-power IGBT's and hard-driven GTO's for high-power inverters," IEEE Trans. Ind. Applicat., vol. 35, pp. 487-495, Mar./Apr. 1999.

[2] H. Stemmler, "High-power industrial drives," Proc. IEEE, vol. 82, pp. 1266-1286, Aug. 1994.

[3] K. Gopakumar, V. T. Ranganathan, and S. R. Bhat, "Split phase induction motor operation from PWM voltage source inverter," IEEE Trans. Ind. Applicat., vol. 29, pp. 927-932, Sept./Oct. 1993.

[4] J. W. Kolar, H. Ertl, and F. C. Zach, "Minimizing the current harmonic RMS value of three-phase PWM converter system by optimal and suboptimal transition between continuous and discontinuous modulation," in Proc. IEEE-PESC Conf. Rec., PESC'91, Cambridge, MA, June 1991, pp. $372-381$.

[5] V. Blasko, "Analysis of a hybrid PWM based on modified space-vector and triangle-comparison methods," IEEE Trans. Ind. Applicat., vol. 33, pp. 756-764, May/June 1997.

[6] A. M. Hava, R. J. Kerkman, and T. A. Lipo, "Simple analytical and graphical methods for carrier-based PWM-VSI drives," IEEE Trans. Power Electron., vol. 14, pp. 49-61, Jan. 1999.

[7] M. Mangal and G. De, "Novel control strategy for sinusoidal PWM inverters," IEEE Trans. Ind. Applicat., vol. 23, pp. 561-566, May/June 1987.

[8] P. G. Handley and J. T. Boys, "Practical real-time PWM modulators-An assessment," Proc. Inst. Elect. Eng. B, vol. 139, no. 2, pp. 96-102, 1992.
[9] J. Holtz, "Pulsewidth modulation for electronic power conversion," Proc. IEEE, vol. 82, pp. 1194-1214, Aug. 1994.

[10] G. Narayanan and V. T. Ranganathan, "Synchronized PWM strategies based on space vector approach. Part 1: Principles of waveform generation," Proc. Inst. Elect. Eng. B, vol. 146, no. 3, pp. 267-275, 1999.

[11] _ "Synchronized PWM strategies based on space vector approach. Part 2: Performance assessment and application to $V / f$ drives," Proc. Inst. Elect. Eng. B, vol. 146, no. 3, pp. 276-281, 1999.

[12] Y. Iwaji, T. Sukegawa, T. Okuyama, T. Ikimi, M. Shigyo, and M. Tobise, "A new PWM method to reduce beat phenomenon in large-capacity inverters with low switching frequency," IEEE Trans. Ind. Applicat., vol. 35, pp. 606-612, May/June 1999

[13] D. G. Holmes, "The significance of zero space vector placement for carrier-based PWM schemes," IEEE Trans. Ind. Applicat., vol. 32, pp. 1122-1129, Sept./Oct. 1996.

[14] S. R. Bowes and A. Midoun, "Suboptimal switching strategies for microprocessor-controlled PWM inverter drives," Proc. Inst. Elect. Eng. B vol. 132, no. 3, pp. 133-148, 1985.

[15] J. Holtz, W. Lotzkat, and A. Khambadkone, "On continuous control of PWM inverters in the overmodulation range including the six-step mode," IEEE Trans. Power Electon., vol. 8, pp. 546-553, July 1993.

[16] D.-C. Lee and G.-M. Lee, "A novel overmodulation technique for space-vector PWM inverters," IEEE Trans. Power Electron., vol. 13 , pp. 1144-1151, Nov. 1998

[17] S. Bolognani and M. Zigliotto, "Novel digital continuous control of SVM inverters in the overmodulation range," IEEE Trans. Ind. Applicat. vol. 33, pp. 525-530, Mar./Apr. 1997.

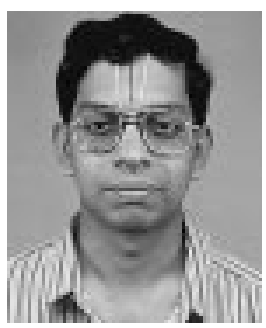

G. Narayanan (S'99-M'01) received the B.E degree from Anna University, Madras, India, in 1992, the M.Tech. degree from the Indian Institute of Technology, Kharagpur, in 1994, and the Ph.D. degree from the Indian Institute of Science, Bangalore, in 2000 .

He is a Research Associate in the Department of Electrical Engineering, Indian Institute of Science. His research interests include ac drives, pulsewidth modulation, multilevel inverters, and protection of power devices.

Dr. Narayanan received the Innovative Student Project Award from the Indian National Academy of Engineering in 2000.

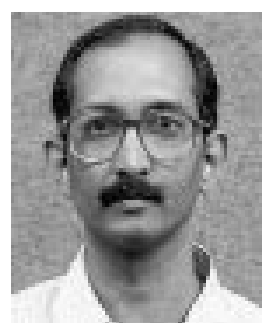

V. T. Ranganathan (SM'84) received the B.E. and M.E. degrees in electrical engineering from the Indian Institute of Science (I.I.Sc.), Bangalore, and the Ph.D. degree from Concordia University, Montreal, QC, Canada.

He joined the Electrical Engineering Department, I.I.Sc., in 1984 and is currently a Professor. His research interests are in the area of power electronics and motor drives. He has published several papers in the areas of vector control of ac drives, PWM techniques, split phase induction motor drives, and rotor side control of slip ring induction motors. He is also a Consultant to industry in the above areas and has participated in a number of projects.

Dr. Ranganathan received the Prize Paper Award of the IEEE-IAS Static Power Converter Committee and the Tata Rao Prize of the Institution of Engineers, India. He is a fellow of the Institution of Engineers, India. 